1 DOSSIER THÉMATIQUE 1

SIUE DEUS SIUE DEA. DÉNOMINATIONS DIVINES DANS LES MONDES GREC ET SÉMITIQUE : UNE APPROCHE PAR LE GENRE

98 DOSSIER THÉMATIQUE 2

TRADITION ET TRANSMISSION DANS L'ANTIQUITÉ : RÉFLEXIONS INTERDISCIPLINAIRES

159 ACTUALITÉ DE LA RECHERCHE

QUOI DE NEUF À L'OUEST DE STRASBOURG ? KOENIGSHOFFEN :

ÉTAT DES LIEUX ET DÉCOUVERTES RÉCENTES

VARIA

195 LuCa BASILE

Forme di contatto, scambio ed interazione culturale nella tradizione vascolare campana tra VIII e VII secolo a.C.

207 Véronique PITchoN

Le faste de la table dans la poésie abbasside

218 Cassandre HARTENSTEIN

La période strasbourgeoise de Pierre Montet (1919-1948)

233 Anna Maria Desiderio et Arianna Esposito

Genre et mobilité à l'aune des relations socio-culturelles : I'exemple de la Campanie archaïque 


\section{GENRE ET MOBILITÉ À L'AUNE DES RELATIONS SOCIO-CULTURELLES : L'EXEMPLE DE LA CAMPANIE ARCHAÏQUE}

\author{
Anna Maria DESIDERIO \\ Ater en Histoire de l'art et archéologie \\ du monde romain, Université Paris Nanterre, \\ UMR 7041 ArScAn - ESPRI, \\ Università degli studi di Salerno, Dispac \\ annamariadesiderio@gmail.com
}

\author{
Arianna ESPOSITO \\ Maître de conférences, \\ Université Bourgogne Franche-Comté, \\ UMR 6298 ARTEHIS - Centre Jean Bérard, \\ USR 3133 CNRS/EFR \\ Arianna.Esposito@u-bourgogne.fr
}

\section{RÉSUMÉ}

À partir des cas d'étude de Pithécusses et de Pontecagnano, I'observation des relations interculturelles et économiques en Campanie à l'aube de la colonisation grecque permet de s'interroger sur la définition et sur la construction sociale du genre au sein de ces deux communautés très ouvertes à des groupes allochtones. Cet article se focalise sur les phénomènes de mobilité et, plus particulièrement, sur la mobilité des femmes. Les cas de Pithécusses et de Pontecagnano illustrent la nécessité de penser une articulation intégrée des

MOTS-CLÉS

Pithécusses,

Pontecagnano, mobilité, pratiques funéraires, genre,

culture matérielle. différentes dimensions sociales, tant au niveau des individus qu'au niveau des structures et des processus sociaux, pour une meilleure compréhension des expériences de mobilité en Campanie archaïque.
Started from cases study of Pithekoussai and Pontecagnano, the observation of intercultural and economic relations in Campania at the dawn of Greek colonization provides an opportunity to review the definition and social construction of gender within these two communities very open to allochtonian groups. This article focuses on mobility phenomena and, in particular, on women's mobility. The cases of Pithekoussai and Pontecagnano illustrate the need to think about an integrated articulation of various social dimensions, as much on the level of individuals as on the social structures and processes, for a better understanding of mobility experiences in archaic Campania.

\footnotetext{
KeYWORDS

Pithekoussai, Pontecagnano, mobility, burial practices, gender, material culture.
} 
À I'heure où la mobilité est une notion incontournable dans notre société, elle s'impose aussi comme un sujet majeur en archéologie [1]. Le renouveau de cette question à travers le prisme de la société contemporaine se développe à un rythme accéléré dans pratiquement toutes les disciplines des sciences humaines et sociales. Certaines réflexions récentes ont eu un impact important et suscité le renouvellement de l'historiographie sur les interprétations des formes et des destinations de ces migrations anciennes [2]. Parallèlement, on constate que ce thème de recherche tend à s'autonomiser pour constituer un domaine d'études à part entière, à l'instar des gender studies, des cultural studies, des subaltern studies et des études postcoloniales.

La migration, volontaire ou forcée, n'est pas un objet scientifique nouveau en soi, mais l'actualité et les nouvelles méthodes révèlent des aspects de son historiographie. Ainsi, il apparaît que les travaux sur ces questions ont longtemps uniquement porté sur les hommes. Artisans, guerriers, mercenaires, marchands... : leur mobilité a été retenue comme un facteur primordial pour la transmission des savoir-faire technologiques et la diffusion de pratiques entre les élites méditerranéennes. Ce paradigme a laissé peu d'espace pour les femmes. L'étude de la mobilité féminine est demeurée ainsi assez marginale. Cette situation cependant commence à changer [3].

L'archéologie postprocessuelle, l'intérêt des chercheurs pour la connectivité et les networks, les global studies, l'importance ainsi que l'imbrication des échelles globales, régionales et locales, les études postcoloniales et l'archéologie du genre, témoignent tous de la diversité des perspectives à travers lesquelles cerner ces mouvements, individuels ou collectifs.

Ces approches, en lien avec le développement de nouvelles méthodes de travail, en particulier de I'anthropo-biologie, ont souligné que la migration et, d'une façon plus générale, la mobilité relèvent d'un processus multiscalaire. Autant le dire d'emblée : au cours de la dernière décennie, les travaux sur la mobilité ont contribué à dessiner un tableau plus dynamique et permis de dépasser l'essentialisme inhérent au concept même de « culture archéologique » [4]. La mobilité est susceptible d'ouvrir des nouvelles perspectives de recherche pour explorer des phénomènes économiques, sociaux, culturels et redessiner la géographie des relations culturelles ou de la circulation des savoirs et des savoir-faire.

Dès lors, il s'agit de mettre l'accent sur les acteurs, les pratiques, les contextes et les réseaux locaux qui ont soutenu les migrations et qui ont permis la mobilité, au sein et entre les communautés, afin de mettre en évidence leurs dimensions sociales et économiques [5]. La mobilité, de fait, ne se réfère pas seulement au déplacement spatial lui-même, mais elle renvoie également au processus d'accueil dans la nouvelle communauté. Dans ce cadre, on porte désormais une attention plus grande à l'individu. Ce dernier est considéré comme un agent [6]. Cette démarche permet de percevoir la fluidité des identités de genre : I'identité de sexe est reliée à d'autres facettes, telles que l'âge, la classe sociale, l'appartenance ethnique.

Ce constat soulève plusieurs questions de méthode. En premier lieu, il souligne la difficulté d'interpréter la culture matérielle en contexte funéraire. Celui-ci est, en effet, par définition, ambigu [7]. Aussi, en l'absence de données biologiques pour identifier le sexe du défunt, les recherches se fondent presque exclusivement sur la reconnaissance d'indicateurs considérés comme genrés (i.e. armes et rasoirs du côté masculin ; accessoires pour la filature ainsi que certaines parures pour le domaine féminin). Cette opposition entre mobiliers masculins et mobiliers féminins suppose implicitement une équivalence entre sexe et genre [8].

[1] Dommelen 2014 ; Desiderio \& Esposito 2020, p. 141-142.

[2] MOATTI 2004 ; MOATTI 2019 ; MOATTI à paraître.

[3] IsAYEV 2017, p. 32-34 ; CASTIGLIONI 2019, p. 98-103 ;

Atti Taranto à paraître $b$. Pour des synthèses problématisées récentes, voir JAMES \& DILLON 2012.

[4] Cf. Reiter \& Frei 2019.

[5] Dommelen 2014 ; BOUfFier 2017.

[6] Sur les apports de l'agency theory à l'interprétation

archéologique, voir KnAPP \& DommeLEn 2008. Pour l'emploi de la notion d'agency dans les études sur le genre, voir HAICAULT 2012.

[7] Voir D'Agostino 1985 ; Cuozzo 2003, chap. 1.2, avec bibliographie; EsPosito \& Pollini 2018, p. 42-43. Voir infra n. 13.

[8] BÉRARD à paraître. Nos remerciements à Reine-Marie Bérard qui nous a donné accès à son article inédit. 
Cependant, les travaux de Mariassunta Cuozzo sur les tombes princières féminines de Pontecagnano (début du VII s. av. J.-C.) ont montré que le statut des défuntes peut s'afficher à travers une appropriation, négociation et réinterprétation d'objets-symboles, idéologiquement chargés, rattachés aussi à la sphère masculine [9]. Autrement dit, ces mêmes femmes possèdent des attributs issus des rapports sociaux. Cet exemple met en évidence la nécessité de contextualiser ces marqueurs dans le régime de genre de la société étudiée. Il faut se détacher des catégories de genre contemporaines et, surtout, ne pas surestimer la place de l'identité de sexe dans l'ensemble des critères qui viennent définir et caractériser une personne. La définition du genre comme outil d'analyse proposée par I'historienne Françoise Thébaud prend tout son sens : « Le sexe est perçu comme un invariant, tandis que le genre est variable dans le temps et l'espace, la masculinité ou la féminité - être homme ou femme ou considéré comme tel(le) - n'ayant pas la même signification à toutes les époques et dans toutes les cultures [10] ».

Le cas célèbre des fibules italiques dans les mobiliers des nécropoles des colonies grecques d'Italie méridionale et de Sicile montre lui aussi les limites de certaines analyses [11]. La présence de ces objets dans les mobiliers funéraires a imposé l'idée de mariages interethniques, sur la base d'une relation directe (et parfois d'un raisonnement circulaire) entre l'origine de la fibule et l'appartenance ethnique du défunt : la présence de ces objets renvoie-t-elle à une identité de sexe ou bien à un statut ou une origine ? Il s'avère que les raisons de la présence de ces artefacts peuvent, dans certains cas, s'expliquer également par le commerce. Les témoignages de Syracuse, en particulier, ont été réétudiés en ce sens. Les chercheurs sont ainsi parvenus à de nouveaux résultats. Pour le site de Mégara Hyblaea, les recherches récentes ont montré que, parmi toutes les catégories d'objets du mobilier funéraire, seules les épingles pouvaient être associées

[9] Cuozzo 2003 ; Cuozzo 2008, p. 110.

[10] THÉBAUd 1998, p. 114. Signalons, dans cette perspective : Boehringer \& Sebillotte Cuchet (dir.) 2011. Cf. également : Boehringer \& Cuchet 2013 ; Boehringer, GRAND-CLÉment, PÉRÉ-NoguÈs \& CUCHET 2020. Ces deux articles comportent des réflexions théoriques sur l'intérêt de l'outil du genre en sciences de l'antiquité, en considérant le genre dans un contexte chronoculturel donné et dans ses interactions avec d'autres formes de différenciations : le statut politique, le positionnement socio-économique et l'ancrage dans la parenté, entre autres. [11] Sur les fibules voir : Hodos 1999, p. 73, pour l'interprétation commerciale ; SHEPHERD 1999, p. 283-285, sur les risques de surinterprétation ; BARTOLONI \& NIZZO 2005, p. 413-423, pour les comparanda et la chronologie ; SHEPHERD 2005, pour la comparaison avec le contexte sicilien ; ibidem, spécifiquement aux défunts de sexe féminin [12]. Chaque cas doit donc être évalué individuellement.

Il faut toujours rester prudent en l'absence de vérification par l'anthropologie biologique. Une sépulture ne reflète pas l'identité personnelle d'un défunt, mais son identité sociale [13]. Une étude sociologique funéraire est certes possible sans données anthropo-biologiques directes, mais à la condition de mener une analyse rigoureuse de l'ensemble du mobilier d'accompagnement et en fonction des données propres à chaque site. La création de nouvelles communautés à Pithécusses, dans le golfe de Naples, et le phénomène de poléogenèse étrusque à Pontecagnano, au sud de Salerne, permettent de s'intéresser à l'identité sociale et culturelle des sociétés autochtones à l'aube de la colonisation grecque en Campanie (fig. 1). À travers ces deux exemples, il est possible de s'interroger sur la construction socio-culturelle du genre au sein de ces deux établissements qui apparaissent, du point de vue de la phénoménologie archéologique, comme des sociétés très ouvertes à des groupes allochtones : à Pithécusses, Grecs, Levantins et indigènes cohabitent. L'établissement est en outre inséré au cœur même des phénomènes d'échange et de transfert - objets, pratiques mais aussi individus - avec les mondes étrusque et campanien, avec la région du Latium et la zone adriatique, avec l'ÆEnôtrie, la Daunie et les aires volsque et ibérique [14]. À Pontecagnano, l'intégration de groupes allochtones semble relever d'un processus distinctif de la communauté autochtone. L'établissement s'impose ainsi comme un observatoire privilégié pour envisager des modèles de mobilité géographique des individus en général, et des femmes en particulier.

La complexité culturelle caractéristique de la Campanie ne se résume pas à une opposition ethnique, mais elle décrit, au contraire, une situation dynamique de peuplement qui désigne un espace de négociations entre les différents acteurs de ces échanges [15]. La prise en compte de la mixité d'un groupe social (hommes et femmes) permet ainsi de mieux comprendre les

p. 217, pour l'interprétation des fibules en tant qu'offrande funéraire ; PÉRÉ-NoguÈs 2008, p. 155-157, pour une synthèse problématisée ; Esposito \& ZURBACH 2010, p. 56-58, sur les différentes interprétations ; SHEPHERD 2012 ; SALTINI SEMERARI 2016, en part. p. 70-80.

[12] BÉRARD 2014 ; BÉRARD 2018 ; BÉRARD à paraître (avec les précautions nécessaires dans l'attente des données anthropo-biologiques).

[13] Pour une synthèse historiographique récente et complète des débats sur l'interprétation des données funéraires antiques et sur les nécropoles comme reflet ou non des sociétés, voir : Nizzo 2015.

[14] Guzzo 2012, p. 516 ; Kelley 2012, p. 245 ; Esposito 2018, p. $168-169$; p. 174-175, avec bibliographie.

[15] Cerchiai 2020, p. 99-100. 


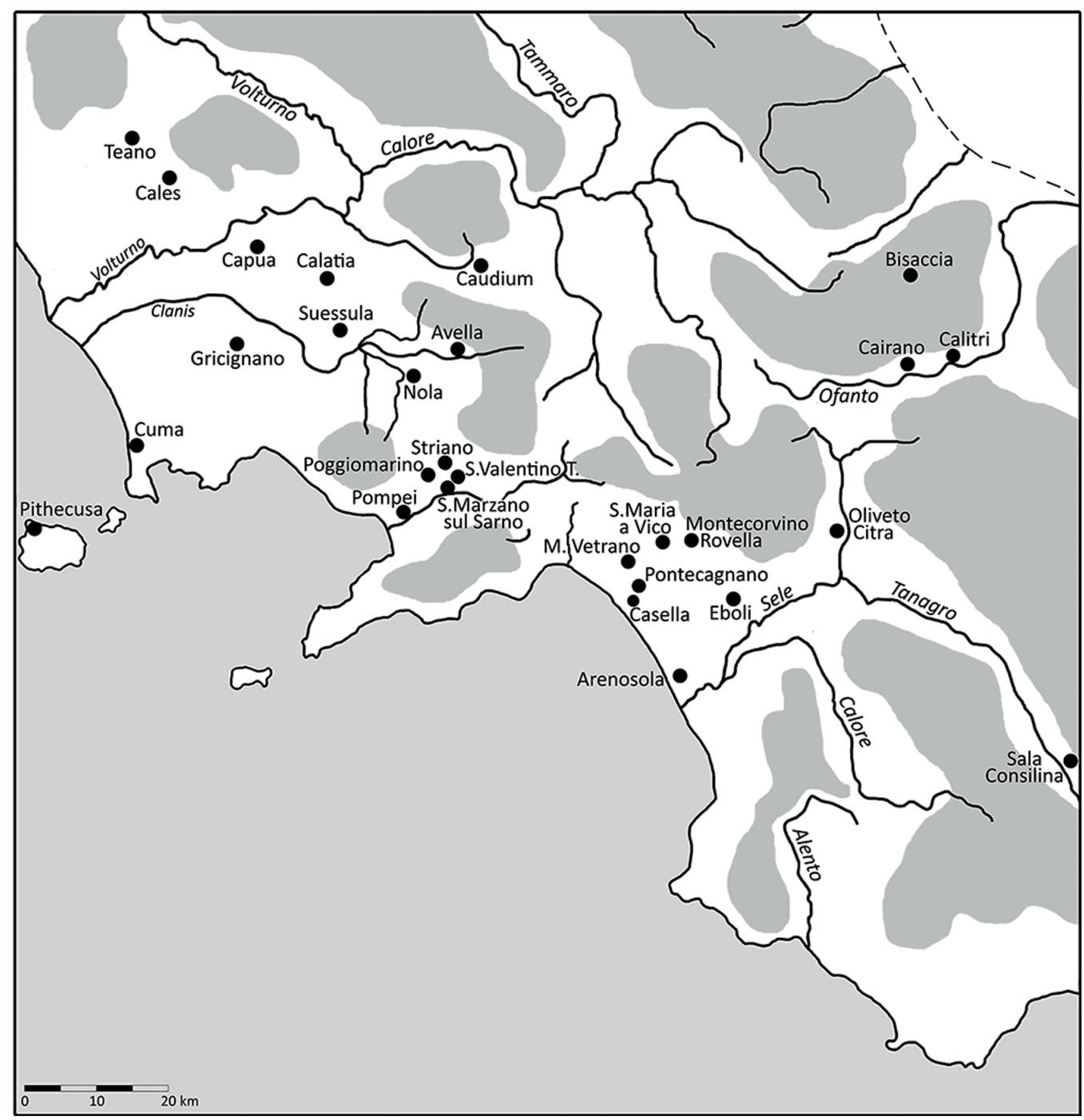

Fig. 1 :

La Campanie aux

VIII - -VII ${ }^{\mathrm{e}}$ s. av. J.-C.

(d'après Pellegrino

et al. 2017,

p. 210 , fig. 1)

phénomènes de mobilité et de mieux percevoir leur complexité. Les femmes sont-elles des agents de liens entre les élites ? Doit-on conjuguer le thème traditionnel des mariages mixtes et de la présence de femmes étrangères à celui des identités ethniques, ou surtout à celui des identités sociales ? La culture matérielle peut-elle suggérer la possibilité de la transmission d'une identité multiple aux enfants?

Les questions soulevées ici portent sur les changements ou le conservatisme dans le registre de la culture matérielle, afin d'explorer la diversité des migrations féminines à Pithécusses et à Pontecagnano. La possibilité d'aborder le sexe biologique et le genre dépend de l'état de la recherche et de la nature de la documentation. Les observations qui suivent devraient permettre d'ébaucher, avec une nécessaire prudence, des hypothèses sur le discours social véhiculé par la culture matérielle [16], en dépit des limites sur la détermination sexuelle des tombes. C'est à la lumière de cette prémisse que cette étude doit être comprise : elle pourra être complétée ou révisée en fonction d'anaIyses anthropo-biologiques futures.

\section{PITHÉCUSSES À TRAVERS LE PRISME DE L'ETHNICITÉ, DU GENRE ET DE L'AGENTIVITÉ}

Nous ne reviendrons pas ici sur le détail des données funéraires pithécussaines ou sur l'histoire de leur interprétation, dont des publications récentes rendent compte [17]. On rappellera juste le caractère manifestement cosmopolite de la communauté, dont la nature multiculturelle se révèle aussi bien dans les diverses pratiques liées au traitement du corps (inhumation, enchytrismos, crémation) que dans les mobiliers funéraires [18].

[16] Sur le rituel funéraire comme occasion de communication sociale : D'AGOSTINo 1985 ; CERCHIAI 2018. [17] Nizzo 2007 ; Cinquantaquattro 2012-2013; Nizzo 2016a ; Nizzo 2016b ; CinquantaquatTro 2017 ; Nizzo 2021.

[18] OsBoRne 2004, p. 24-25 ; Guzzo 2012 ; Nizzo 2016b, p. 427-428; GigANTE et al. à paraître $a$; GigANTE et al. à paraître $b$. 
Les résultats de l'analyse des isotopes du strontium $\left({ }^{87} \mathrm{Sr} /{ }^{86} \mathrm{Sr}\right)$ confirment la présence d'individus nés ailleurs (environ $22 \%$ ), tous des adultes, renforçant l'idée que Pithécusses était une communauté multiethnique [19]. Les dernières recherches sur les lots funéraires publiés dans Pithekoussai I, qui correspondent aux phases de la première utilisation de l'espace funéraire (VIII ${ }^{e-V I}{ }^{e} s$. av. J.-C. : la phase la plus intense), et sur les sépultures encore inédites étudiées lors des fouilles de Buchner 1965-1982 (Pithekoussai II), recourent aux analyses ostéologiques et isotopiques [20]. L'analyse de la proportion statistique montre une légère prépondérance du nombre d'individus masculins adultes étrangers par rapport aux femmes [21], ce qui pourrait être un indice d'unions interethniques. La capacité d'inclusion de cette nécropole suggère que l'absence de femmes inhumées n'est pas la manifestation de gestes funéraires codifiés de type sélectif. Cette absence est probablement due au taux élevé d'adultes dont le sexe biologique n'a pas été déterminé [22].

Les recherches menées par Teresa Cinquantaquattro pointent l'existence d'un modèle communautaire dans lequel l'élite grecque rassemble autour d'elle les différentes composantes culturelles [23]. Les articulations sociales sont transversales et dépassent les critères ethniques [24]. Mais la crémation est réservée aux défunts qui ont été, pleno iure, membres de la communauté, tandis que l'inhumation est le rite de la marginalité, de l'insertion imparfaite dans la communauté de Pithécusses [25].

L'étude menée par Pier Giovanni Guzzo a démontré que dans plus de $50 \%$ des mobiliers funéraires contenant des fibules en bronze, au moins une paire est présente : «a questa percentuale si aggiunge la totalità di quelli con 3 fino a 20 esemplari, nei quali sono coppie, in alcuni casi più coppie, formate da fibule dello stesso tipo. Pertanto, I'uso di indossare almeno una coppia di fibule pertinenti allo stesso tipo appare essere stato proprio del costume personale pithecusano [26] ». D'après cette analyse, la population féminine est celle qui, dans une proportion dominante, utilise des fibules. La plupart de ces attestations concerne des individus non adultes. L'utilisation de ces objets chez les adultes est de fait très faible. Une telle différence entre les femmes et les hommes est attestée aussi dans le cas des fibules en argent qui toutes relèvent de sépultures féminines.

Cette distribution entre classes d'âge pourrait vraisemblablement souligner la valeur culturelle accordée à l'usage des fibules, « in quanto le fibule sono accessorio necessario al funzionamento dell'abbigliamento » dans les mœurs locales [27]. Ces fibules sont ainsi un élément caractérisant l'identité culturelle indigène pithécussaine : un élément qui toutefois se modifie et se raréfie à la fin du VII s. av. J.-C.

La majorité des fibules sont locales. Les liens formels avec des spécimens de Sicile, de Calabre, de Basilicate et des Pouilles s'expliquent en fonction des réseaux d'échanges entre ces zones culturelles au cours du VIII' s. av. J.-C. L'utilisation de fibules, attestée dans 132 lots de mobiliers, soit environ $20 \%$ du total des sépultures publiées, représente un pourcentage qui, s'il n'est pas faible, demeure certainement minoritaire. Selon P. G. Guzzo, une telle proportion peut être comprise comme un signe d'une culture prédominante qui, elle, n'utilise pas de fibules, par opposition au milieu italique indigène dans lequel ces objets sont essentiels à I'habillement. Dès lors il déduit qu'au VIII' $s$. av. J.-C., une partie de la population de Pithécusses est d'origine indigène, surtout campanienne, et principalement féminine [28].
[19] Communication orale : Gigante M., Warter V., Müller W., Sperduti A., Bondioli L. 2016, « Among the Greeks, Among the Natives: Strontium isotopic ratio analysis of the human odontoskeletal remains from Pithekoussai, Ischia (Southern Italy) », Seventh Conference of Italian Archaeology, Galway (Irlande), 16-18 avril 2016, et GIGANTE et al. à paraître. Pour l'interprétation des analyses anthropo-biologiques à Pithécusses, cf. Coldstream 1994, p. 51 ; BeCKER 1995 , p. 273-276 ; SHEPHERD 1999, p. 276 ; GigANTE et al. 2012-2013; GIGANTE et al. à paraître $a$; GIGANTE et al. à paraître $b$.

[20] L'étude anthropo-biologique a porté sur le réexamen de 96 crémations et 12 inhumations, parmi les tombes publiées dans Pithekoussai I, et sur 34 crémations et 61 inhumations inédites, relatives aux fouilles Buchner 1965-1982 : cf. GIGANTE et al. à paraître $b$.

[21] BeCKer 1995 ; Gigante et al. 2012-2013 ; Nizzo 2021, en part. n. 13 ; GIGANTE et al. à paraître $a$; GIGANTE et al. à paraître $b$. Pour les précautions relatives aux mariages mixtes : BÉRARD à paraître.
[22] Cinquantaquattro 2013, p. 69. Pour l'évolution du ratio H/F, voir Nizzo 2007, p. 25-28, fig. 6a-c et les annexes 1 (p. 177-190) et 2 (p. 191-196) ; Nizzo 2021.

[23] CinquantaquatTro 2012-2013; Cinquantaquattro 2017.

[24] DeSIDERIO \& ESPOSITO 2020, p. 144-145.

[25] D'Agostino 2011, p. 40 ; Gigante et al. 2012-2013 ; Nizzo 2021, p. 70-71.

[26] Guzzo 2012, p. 510-511 : « Dei più che 600 individui finora documentati nella necropoli di San Montano, 132 sepolture hanno corredi contenenti fibule in bronzo: circa il $21 \%$. Di questi ultimi 87 sono femminili; 22 maschili; 17 di genere non identificato » (p. 510). Cf. également Hodos 1999, p. 69.

[27] Guzzo 2012, p. 520.

[28] « Tali individui indigeni appaiono essere stati in maggioranza di genere femminile: così indirizza a ritenere la identificazione del massimo numero dei corredi sepolcrali contenenti fibule, ai quali si aggiungono quelli di maschi non adulti, evidentemente composti nella tomba dalle rispettive madri secondo il proprio modello culturale. » (Ibid. p. 520). 
Concernant les fibules comme attribut typiquement féminin, plusieurs précautions doivent être retenues, selon deux explications, par ailleurs non exclusives : d'une part, seule une très modeste portion de la nécropole a été fouillée (10 \%) ; d'autre part, le dépôt des fibules peut aussi relever de l'offrande funéraire, notamment lorsqu'on les retrouve dans les tombes d'enfants. En d'autres termes, à défaut de données anthropo-biologiques sûres, il faut à notre avis reprendre cette question avec une grande prudence. Nous devrions tenir compte de l'intentionnalité des objets déposés et, dans la mesure du possible, d'autres critères également : les croyances ou les coutumes par exemple [29]. En effet, l'identité sociale du défunt doit être reconstituée en considérant la tombe et son mobilier comme un ensemble inséré dans un système plus large, la nécropole, dont il faut restituer à la fois I'organisation et la hiérarchisation. En l'état actuel des publications, s'il est difficile d'affirmer que ces individus appartenaient à l'élite pithécussaine, on peut du moins envisager qu'il s'agirait d'individus allogènes agrégés aux familles dominantes.

À cet égard, on peut davantage essayer de nuancer notre analyse en considérant les données céramiques et en suivant I'hypothèse avancée par Luca Cerchiai, à la suite de Bruno d'Agostino : au sein des family plots, quelques personnages féminins non-Grecs, exceptionnellement incinérés, ont pu avoir un rôle important au sein de la communauté [30]. Ce rôle ne s'exprime pas dans la portée limitée de la seule sphère familiale/ethnique. Ces femmes ont joué un rôle central dans la permanence et la transmission de la culture autochtone (fig. 2). À l'interface entre statut et genre - entendus en termes de représentation sociale -, elles expriment leur identité en choisissant délibérément de mélanger les traditions propres à leur origine italique (les vases en impasto, locaux ou de tradition régionale) avec celles d'ascendance grecque et pithécussaine (le rite de l'incinération, la présence d'un set cohérent de céramiques grecques).

Les femmes de Pithécusses étaient intégrées à un réseau de relations familiales certes, mais aussi sociales et culturelles. Elles ont ainsi contribué à la codification de nouvelles stratégies funéraires symboliques au sein d'un milieu fortement hybride, métissé. Dans le contexte des groupes émergents caractérisés par l'incinération, un groupe restreint de femmes révèle ainsi une dialectique complexe entre conservatisme et innovation. Ces femmes contribuent au partage d'un modèle idéologique commun, interne à leur groupe : «... se si incrociano $\mathrm{i}$ dati relativi alla distribuzione della produzione di impasto locale o di tipo regionale nelle tombe femminili ad incinerazione e nelle sepolture di bambino all'interno dei family plot, si mette in luce una rappresentatività della comunità indigena più articolata di quanto si sia finora supposto perché non ristretta alle sole sepolture ad inumazione all'esterno dei plessi familiari: si individua una ristretta componente femminile contraddistinta dall'uso elitario della cremazione, cui si associa la tendenza a marcare in senso materno i figli premorti - infanti o bambini in tenera età - attraverso la deposizione di un oggetto di impasto [31] ».

L. Cerchiai a comparé cette situation à un contexte indigène voisin et contemporain, Gricignano d'Aversa. Ici, les mobiliers céramiques indigènes en impasto persistent et prévalent. Si l'on suit cette lecture, on peut alors se rendre compte du sens véhiculé par la sélection intentionnelle établie par quelques femmes autochtones de Pithécusses. Elles opèrent ce que Gillian Shepherd a appelé un code switching - concept emprunté à la linguistique pour expliquer la sélection, le rejet ou les combinaisons de traits spécifiques de la culture matérielle offrant des solutions non seulement pour revendiquer un lien et une cohésion de nature sociale, mais aussi pour affirmer la distinction et la séparation [32]. À Pithécusses la communauté grecque semble tenir un rôle dominant. Les mobiliers composites de ces femmes affichent une forme de silent resistance. Cette résistance disparaît à la suite de l'essor de la cité coloniale de Cumes : l'émergence de nouveaux groupes brouille alors les codes sur lesquels se fondait la société précoloniale [33].

\section{PONTECAGNANO : MOBILITÉS ITALIQUES ET RELATIONS DE POUVOIR DANS LA CITÉ ORIENTALISANTE}

Si à Pithécusses les marqueurs liés au monde indigène se concentrent surtout dans les tombes de femmes et d'enfants, dans le centre de culture étrusque de Pontecagnano on observe une situation plus complexe. En effet, de nombreuses études sur les nécropoles orientalisantes (VIIIe-VII $\mathrm{s}$. av. J.-C.) ont livré l'image d'une communauté « ouverte » qui intègre plusieurs composantes de peuplement pendant le processus d'urbanisation.
[29] Par exemple, Hodos 1999, p. 67, considère les fibules davantage comme des indicateurs de classe, de statut ou d'âge que comme des marqueurs de l'ethnicité ou de I'identité. Cf. également BÉRARD à paraître.

[30] D'Agostino 1999, p. 60-62; Cerchiai 2017, p. 232-233 ; Esposito 2018, p. 174-175.

[31] CeRChiai 2017, p. 233.

[32] SHEPHERD 2014, p. 126.

[33] Cerchiai 2017, p. 238. 


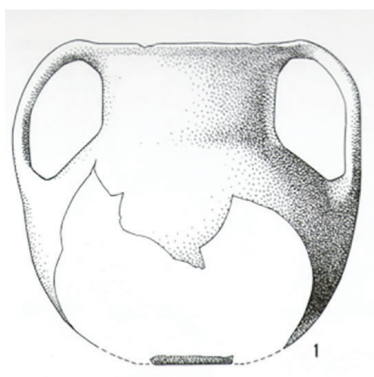

631
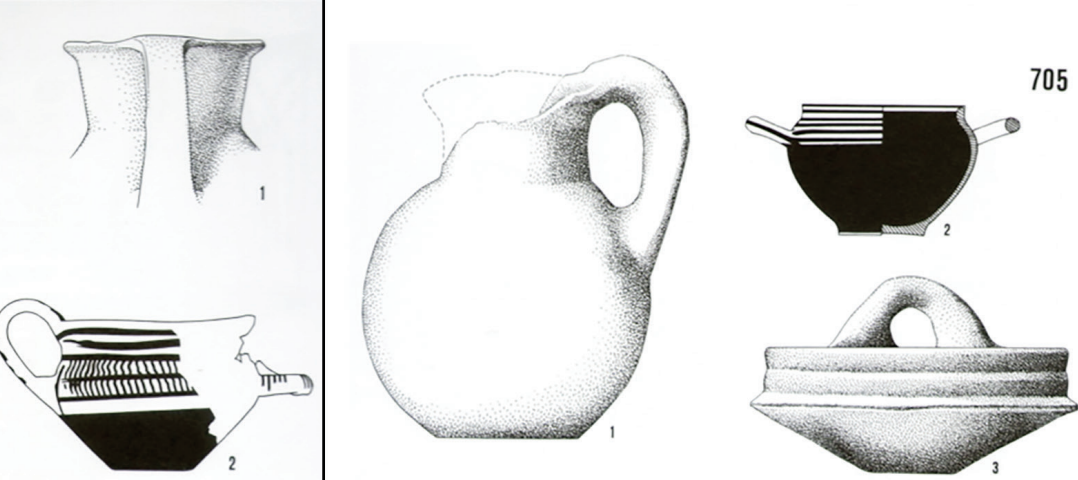

705

A
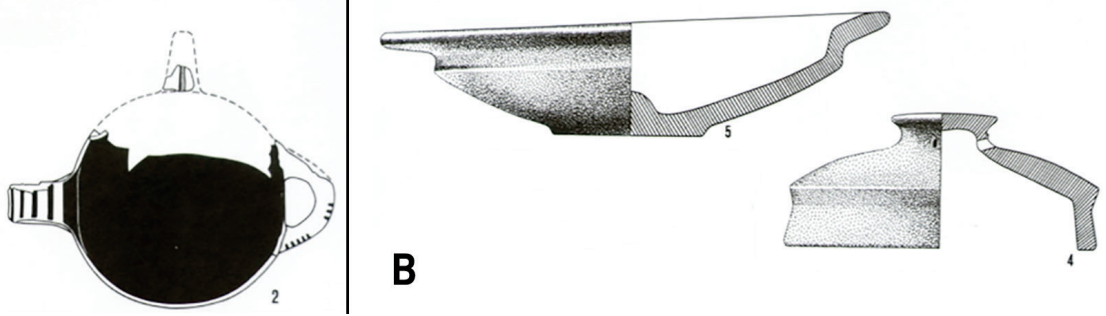

Fig. 2 : Mobiliers en impasto de Pithekoussai. A. T. 631 ; B. T. 705 (d'après Cerchiai 2017, fig. 11 et 14)

Leur inclusion dans la société est favorisée par le mouvement général de restructuration sociale, politique et territoriale qui marque le passage entre le premier âge du fer local et l'époque orientalisante (deuxième moitié du VIIIe s. av. J.-C.) [34].

L'abandon des nécropoles de l'âge du fer en faveur de nouvelles aires funéraires témoigne de ce changement radical. Ces dernières comportent plusieurs noyaux de nature familiale. La pleine visibilité funéraire des enfants - auparavant sous-représentés - indique une nouvelle conception des liens de descendance des groupes et de leur continuité lignagère [35].

Le renouvellement idéologique est sensible aussi dans les formes de représentation funéraire, fondées sur l'adoption généralisée d'un service céramique qui réélabore activement les pratiques grecques de consommation et d'offrande du vin. Cette dotation de base comprend une amphore en impasto locale associée à un vase à verser et à un vase à boire de type grec (œnochoé, skyphos/kylix), ainsi qu'à un récipient pour l'offrande et/ou la consommation du repas solide (coupe/écuelle/plat) [36].

[34] Bonaudo et al. 2009, p. 172-178; Pellegrino 2015, p. 35 ; Cuozzo \& Pellegrino 2019, p. 141-145.

[35] Les secteurs funéraires du premier âge du fer sont des groupements vastes, comprenant plusieurs dizaines de tombes d'adultes et un nombre limité de tombes d'enfants. Au sein des secteurs funéraires implantés dans le dernier quart du VIII $s$. av. J.-C., agencés de manière plus éparse, les tombes s'ordonnent selon des critères moins rigoureux, formant des noyaux différents selon l'extension, I'organisation spatiale et la composition. Ils comportent un petit nombre de sépultures d'adultes, auxquelles s'ajoutent les tombes
Dans cette dimension communautaire, impliquant des normes de représentation funéraire partagées à l'échelle collective, on observe une grande variabilité. Elle relève en partie des stratégies d'autoreprésentation développées par les différents groupes qui composent la communauté, mais également de réelles différences en ce qui concerne leurs ressources économiques ainsi que leur composition sociale [37].

À cet égard, la récurrence de marqueurs matériels précis, tant dans les rituels adoptés que dans les mobiliers funéraires, suggère l'intégration de composantes culturelles externes. Initialement, ces phénomènes de mobilité ont été expliqués par l'hypothèse d'échanges matrimoniaux, en raison de la visibilité immédiate des sépultures féminines avec des parures complexes, étrangères au répertoire local [38]. Les travaux de M. Cuozzo sur la nécropole orientale de S. Antonio ont impliqué un changement de perspective et montré que les échanges matrimoniaux ne sont qu'une des formes d'intégration possibles, parmi d'autres. En effet, dans le noyau sud-ouest de I'aire Chiancone IV, pendant la première moitié du VII $\mathrm{s}$. av. J.-C., on constate la présence d'un groupe homogène du faciès culturel d'Oliveto

des sujets de classes d'âge inférieures, correspondant à $75-80 \%$ de chaque noyau, avec un grand nombre d'enfants décédés dans les premières années de leur vie. La pleine représentation funéraire des enfants est une anomalie par rapport à d'autres communautés protohistoriques tyrrhéniennes contemporaines : Cuozzo 2003, p. 203-211; Pellegrino 2015, p. 36 ; Cuozzo \& Pellegrino 2019, p. 141. [36] Cuozzo 2003, p. 196-197.

[37] Cuozzo \& Guidi 2013, p. 83 ; Cuozzo \& Pellegrino 2019, p. 147.

[38] Cerchiai 1995, p. 93-94. 


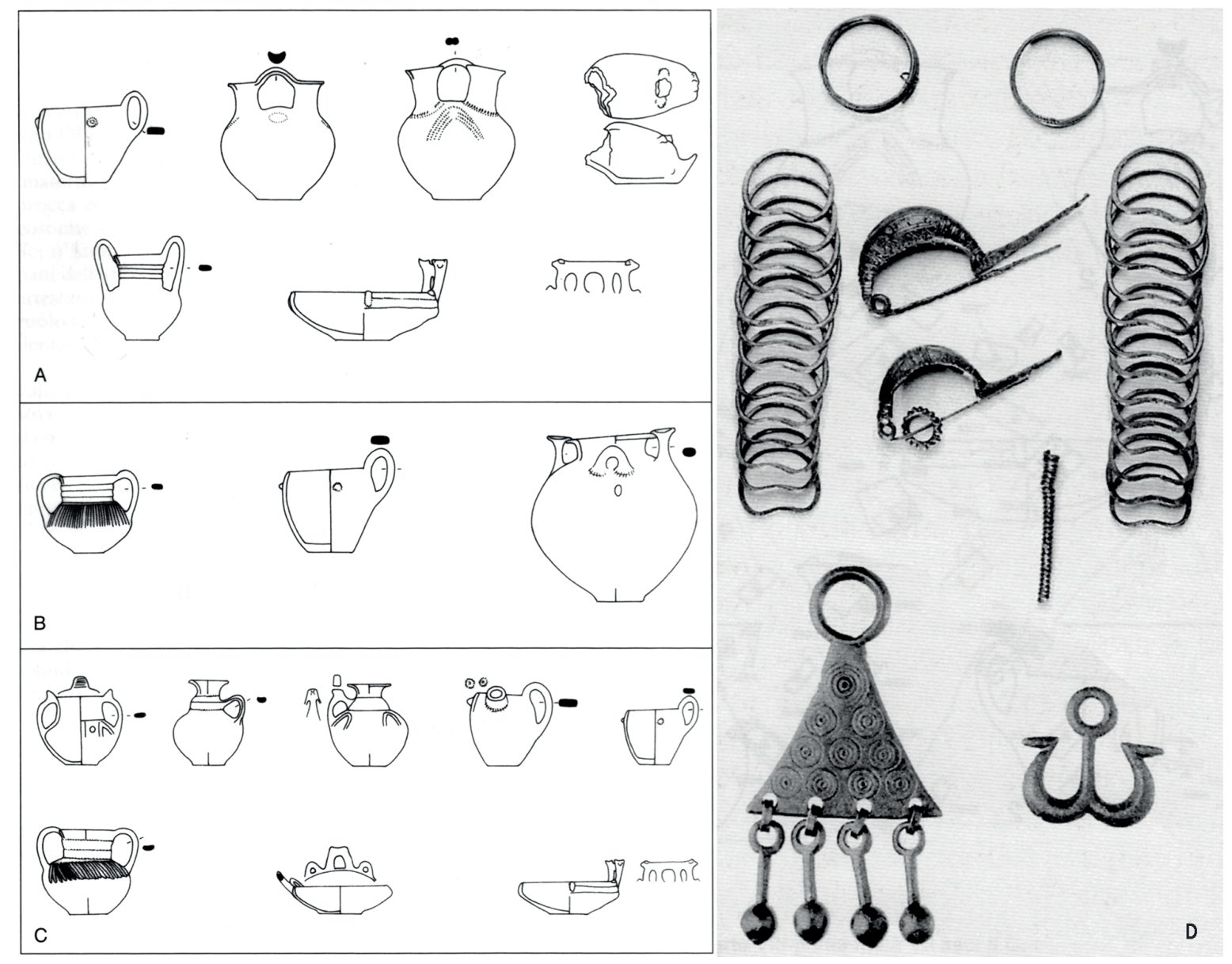

Fig. 3 : Pontecagnano, nécropole orientale de S. Antonio. A-C. Les services céramiques du faciès de Oliveto Citra - Cairano ; D. Les ornements du faciès de Oliveto Citra - Cairano (d'après Cuozzo 2000, fig. 7-8)

Citra - Cairano, caractérisant les sites des vallées fluviales de I'Ofanto et du Sele [39].

Cette composante culturelle est reconnaissable par les ornements féminins, parmi lesquels les bracelets à arc infléchi, les pendentifs triangulaires, en forme d'oméga ou de goutte, les boucles d'oreilles aux extrémités spiralées. Par ailleurs, certains vases spécifiques en impasto, comme les amphores à anses complexes ou surmontantes, le bol à anse en croissant lunaire, le bocal, la cruche à lèvre évasée, l'askos ont une valeur de véritable marqueur culturel [40]. (fig. 3A-D)

Les éléments de la culture matérielle d'Oliveto Citra Cairano caractérisent de manière plus flagrante les sépultures de femmes et d'enfants, mais se retrouvent également dans les tombes masculines, permettant ainsi d'inférer la mobilité d'un groupe entier d'individus. L'adhésion aux normes de représentation funéraire collective, à travers l'adoption du mobilier de base, et les hauts niveaux d'ostentation montrent que le groupe est intégré à la communauté dans une position sociale non subalterne. L'accentuation de traits spécifiques de la culture matérielle d'Oliveto Citra - Cairano est une expression de la stratégie funéraire adoptée, visant à créer une identité alternative à celle qui est dominante.
Dans cette dynamique de promotion sociale, ouverte et compétitive, les différents groupes détenteurs des aires funéraires s'opposent et se confrontent.

M. Cuozzo a souligné l'imbrication des dynamiques ethniques dans la construction de l'identité de genre et de statut de la composante féminine via la culture matérielle. Le caractère éminent des tombes féminines du secteur - avec les outils en fer liés à la sphère du foyer et du sacrifice - et leur lien direct avec les sépultures d'enfants jusqu'à deux ans - explicité par la récurrence des mêmes formes céramiques - montrent la place accordée à la femme, garante de la continuité du groupe. Il s'agit d'un comportement funéraire caractéristique de la nécropole orientale, soulignant le rôle central des femmes dans les systèmes de descendance [41].

[39] Cuozzo 2000, p. 344-349 ; Cuozzo 2003, p. 133-167 ; p. 219-223 ; Cuozzo \& Guidi 2013, p. 83-86.

[40] Cuozzo 2000, p. 341 et fig. 7-8; Cuozzo 2003, p. 67 ; Cuozzo \& Guidi 2013, p. 85.

[41] En effet, elles se trouvent souvent en position centrale dans l'espace funéraire et sont accompagnées d'indicateurs de prestige - chenets, broches/couteau, hache - qui suggèrent leur fonction de garantes du foyer et du groupe : Cuozzo 2003, p. 212-218; p. 231. 


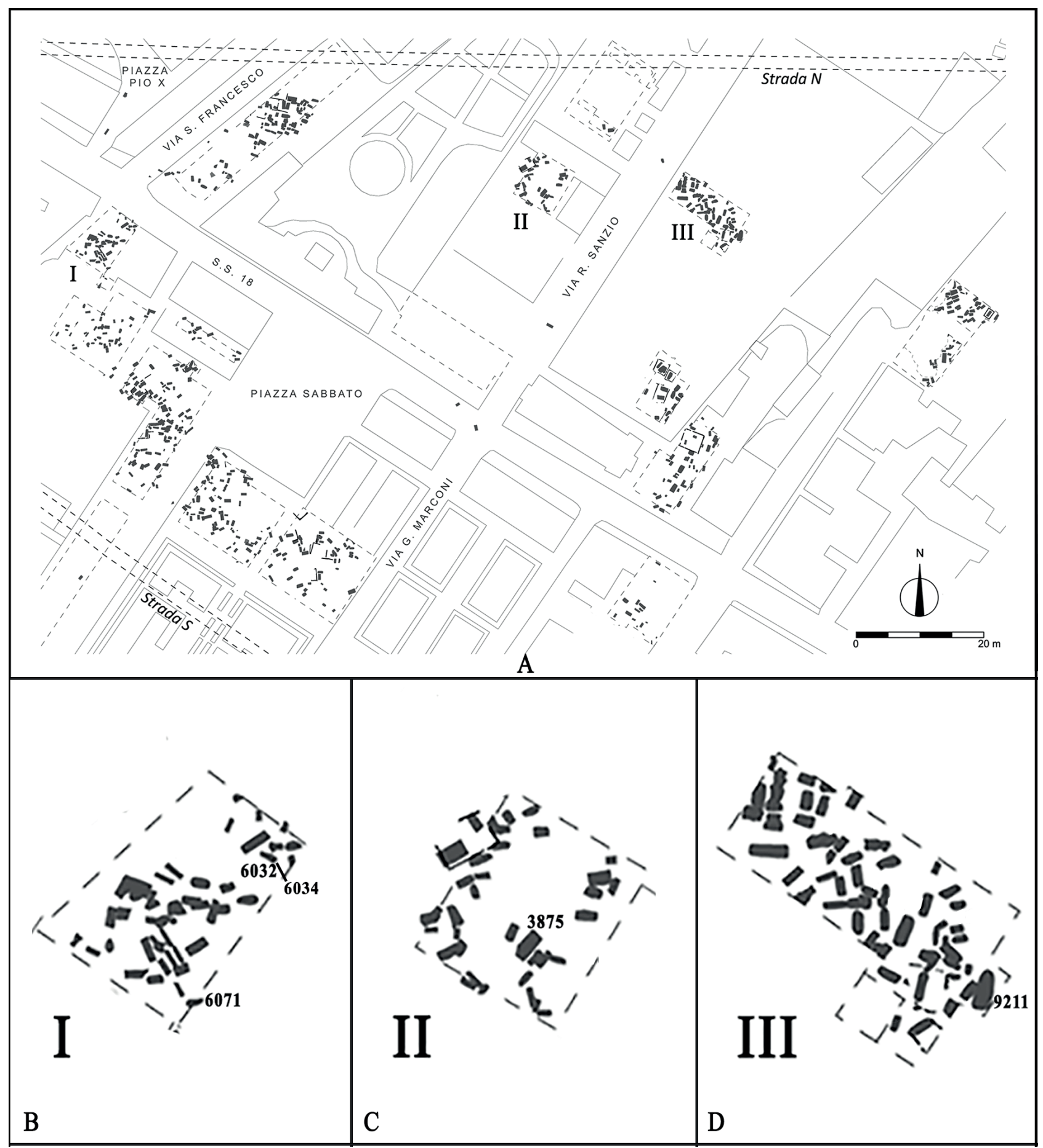

Fig. 4 : Pontecagnano, les aires funéraires de la nécropole occidentale de Piazza Sabbato (réélab. d'après Pellegrino et al. 2017, p. 227, fig. 9)

Les noyaux funéraires du secteur Piazza Sabbato de la nécropole occidentale révèlent une situation différente (fig. 4A), avec des phénomènes de mobilité hétéroclite. Ces aires funéraires accueillent des éléments issus de plusieurs réalités régionales du monde italique, expression d'un large système de relations et de mobilités qui intègre les diverses composantes installées le long des Apennins [42]. Par ailleurs, leur intégration n'a pas un caractère occasionnel, puisqu'elle est manifeste, dès la fin du VIII ${ }^{e}$ s. av. J.-C., pour deux ou trois générations, jusqu'au troisième quart du VII $\mathrm{s}$. av. J.-C [43].
La grande variété des comportements funéraires adoptés par les composantes allogènes soulève la question de l'existence de différentes modalités et degrés d'intégration au sein de la société de Pontecagnano [44].

[42] Pellegrino et al. 2017, p. 232-233; Pellegrino \& Rizzo 2018, p. 155.

[43] Cerchiai 2013, p. 150 ; Cerchiai et al. 2013, p. 81 ; Pellegrino et al. 2017, p. 225 ; Pellegrino \& Rizzo 2018, p. 154.

[44] Pellegrino 2015, p. 43 ; Cuozzo \& Pellegrino 2019, p. 148. 
Considérons, tout d'abord, l'insertion des éléments étrangers dans les divers noyaux funéraires de la nécropole. Ils peuvent être isolés à l'intérieur de groupes où il n'y a pas d'autres individus allochtones. À l'inverse, ils s'agrègent dans les cas où toutes les composantes démographiques du groupe funéraire affichent une origine allogène. Cette opposition est l'indice de formes de mobilités individuelles ou collectives. Mais elle pose aussi la question du possible rôle intermédiaire des groupes locaux dans l'intégration des étrangers.

Le cas de deux femmes de plus de 50 ans en position recroquevillée (TT. 6032 et 6071) dans le secteur funéraire Gaeta (I, fig. 4B ; fig. 5A), au début du VII $\mathrm{s}$. av. J.-C. - le rituel et les associations des mobiliers les rattachent à la moyenne vallée de I'Ofanto - semble s'inscrire dans cette perspective [45]. L'âge avancé des deux femmes et leur position isolée dans le groupe - dépourvu d'autres présences allogènes et vraisemblablement caractérisé par une sous-représentation $d$ 'hommes adultes - suggèrent leur inclusion dans un cadre autre que celui du conubium impliquant probablement des rapports de subordination ou de dépendance [46].
Des niveaux d'intégration sensiblement différents définissent en revanche deux groupes d'origine allogène, implantés dans les secteurs Biblioteca et Promenade archéologique (II et III, fig. 4C et D). Ils comportent des individus inhumés avec des mobiliers céramiques ou des parures de type Oliveto Citra - Cairano, agrégés à des défunts accompagnés par des indicateurs matériels qui renvoient à la vallée du Sarno ou à la Campanie septentrionale, à l'aire nord-lucanienne ou médio-adriatique [47].

Les deux révèlent, en outre, que le processus d'intégration peut se réaliser à différents niveaux de l'échelle sociale. Les caractères les plus éminents se concentrent dans les sépultures de femmes adultes [48]. C'est le cas des tombes 3875 (Biblioteca) et 9211 (Promenade) - des débuts du VII s. av. J.-C. - qui se distinguent respectivement par une lance utilisée comme marqueur et par un petit tumulus. Les deux femmes sont accompagnées par des indicateurs de rôle et de fonction, la fusaïole et l'arme pour la T. 3875, les broches en fer pour la T. 9211. Leurs parures de bronze comportent les éléments caractéristiques de la culture d'Oliveto Citra - Cairano. Dans la T. 9211, le niveau éminent de

\section{Fig. 5 :}

Secteur Gaeta A.

T. 6032 (d'après

Cinguantaguattro \&

Cuozzo 2002,

p. 133 , fig. 6$)$;

B. œnochoé en impasto

de la T. 6034 (d'après

CinguantAquatTRO

2004-2005,

p. 159 , fig. 7)
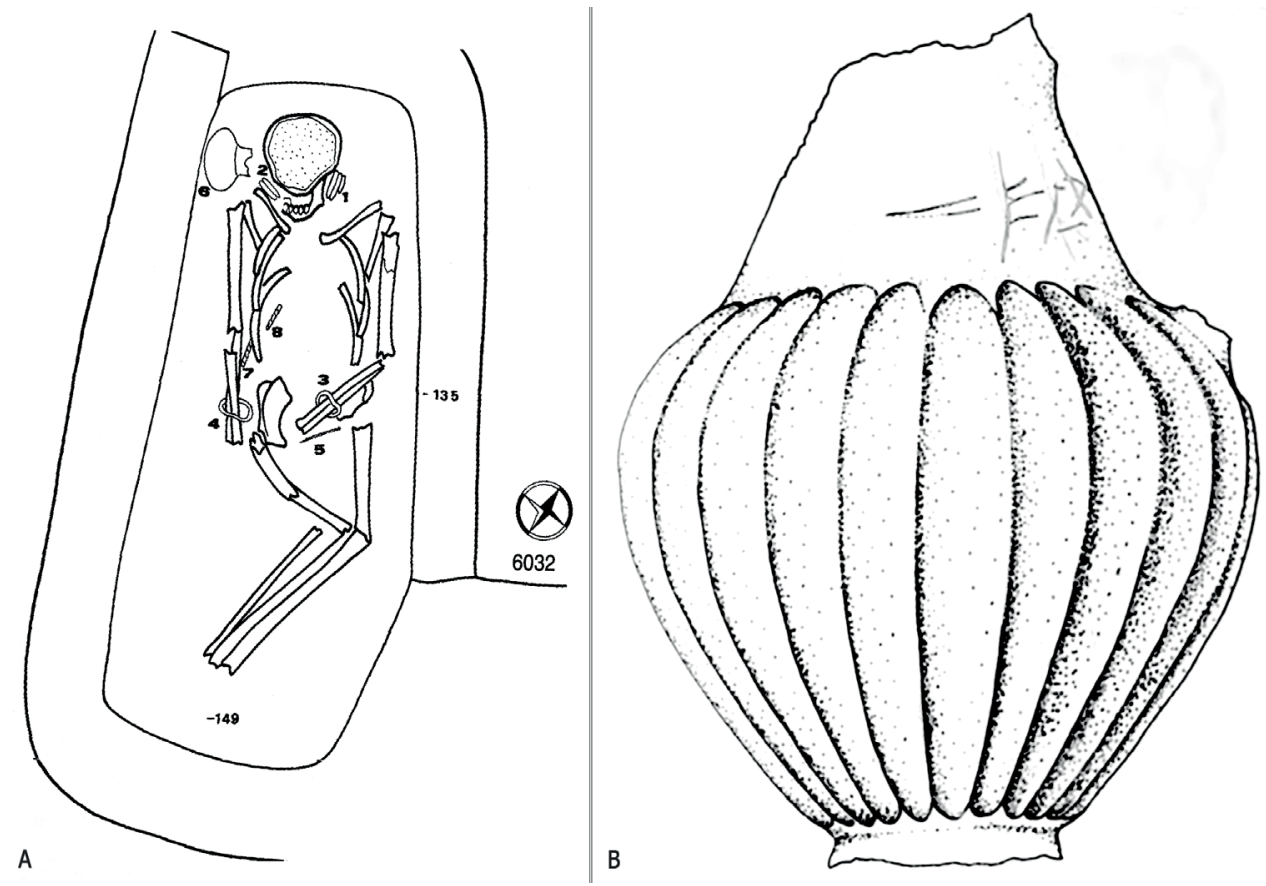

[45] Le rituel de I'inhumation du corps en position recroquevillée est inhabituel à Pontecagnano. Il se rattache plutôt à une vaste zone géographique qui s'étend principalement à l'est de l'Apennin, sur l'aire adriatique et ionienne, comprenant le Picénum, la Daunie et la Basilicate orientale : CinquantAQUATTRO \& CuOzzo 2002, p. 132-134 ; pour la région picénienne, voir : D'ERCOLE 2002, p. 331, n. 126 (avec bibliographie précédente).

[46] Pendant la première phase d'occupation du secteur, datée du début du VII $\mathrm{s}$. av. J.-C., on constate que le rapport entre hommes et femmes adultes n'est pas équilibré (1 sur 3). Pour la détermination du sexe et de l'âge des défunts du secteur funéraire : CinquantaquaTtro 2004-2005, p. 155-157, n. 5. [47] Pellegrino et al. 2017, p. 227-234 ; p. 249-258; Pellegrino \& Rizzo 2018, p. 154-155; Desiderio \& Esposito 2020, p. $163-165$.

[48] Dans cette perspective, le rôle social des femmes apparaît similaire à celui observé dans les centres de la mesogaia campanienne, en particulier à Avella, où I'ostentation des ressources économiques des groupes prééminents peut caractériser à la fois des hommes et des femmes : CinguANTAQUATTRO 2006-2007. 

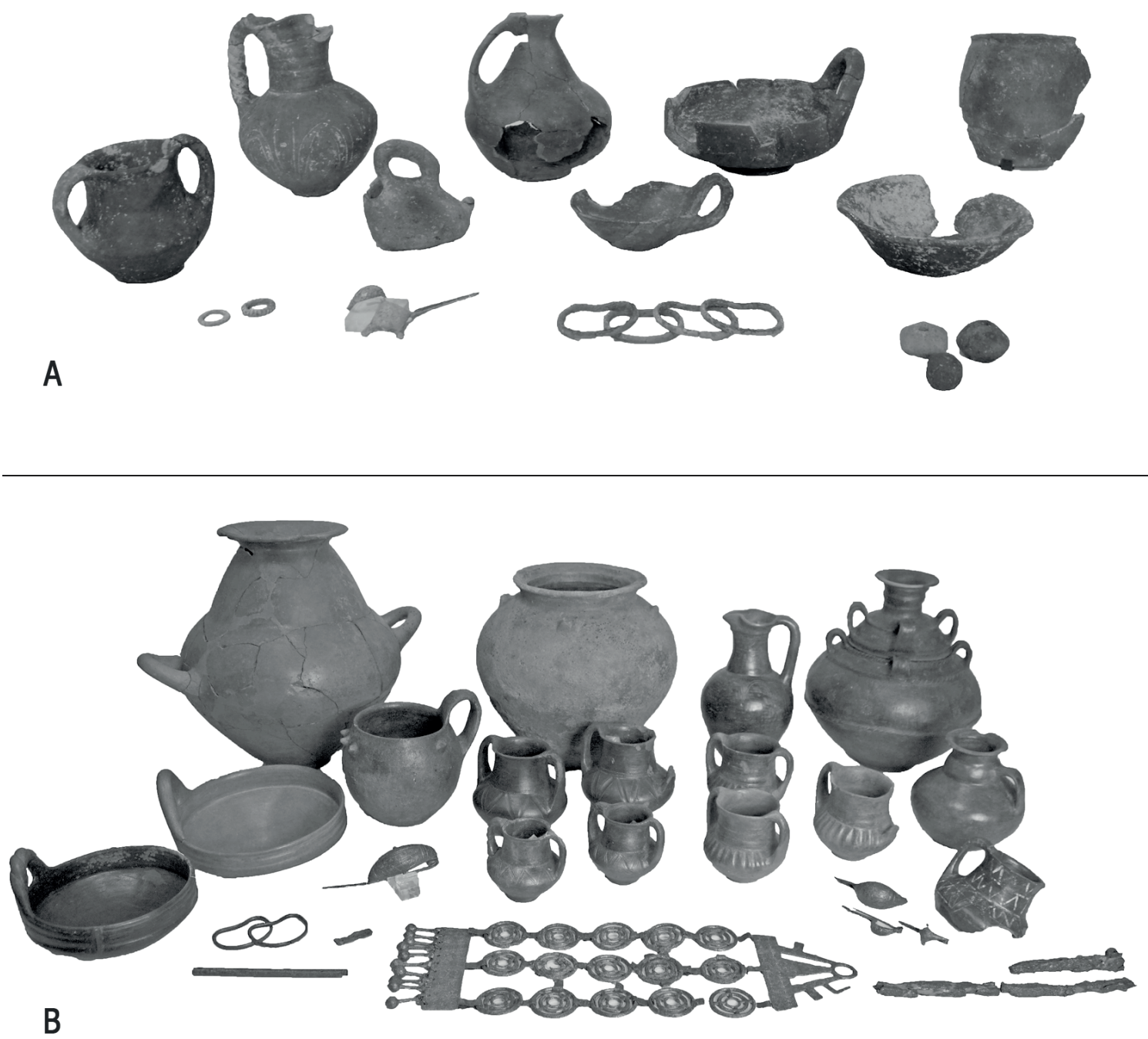

Fig. 6 : A. Sélection du mobilier de la T. 3875 ; B. Mobilier de la T. 9211 (réélab. d'après DeSiderio 2018, p. 75, fig. 4)

la défunte est également marqué par une pendeloque exceptionnelle, portée à la taille. Elle rassemble, dans une composition originale, plusieurs éléments hétérogènes, issus de différentes aires culturelles d'Italie méridionale et probablement associés progressivement. Les mobiliers céramiques intègrent des types de production locale et des formes propres à différents milieux culturels indigènes de Campanie, notamment à la culture d'Oliveto Citra - Cairano, mais également à la vallée du Sarno.

Les assemblages des deux sépultures (fig. 6A-B) montrent, cependant, des différences dans la réception de la norme du mobilier de base, absent de la riche T. 9211 et présent, en revanche, dans la T. 3875. Cette sélection révèle le processus d'appropriation active des pratiques rituelles locales de la part des défuntes des TT. 3875 et 9211 , que I'on retrouve également chez leurs groupes d'appartenance [49].
Les deux noyaux allogènes sont ainsi caractérisés par une large variété de comportements funéraires, expression d'une opposition entre le choix du rite ou d'une culture matérielle indigène et l'acceptation progressive des normes locales de représentation funéraire [50].

L'existence de plusieurs niveaux d'adéquation aux normes communautaires met en évidence la dynamique d'interaction qui s'établit entre les comportements collectifs et ceux de groupes particuliers. De plus, les différents choix de représentation funéraire suggèrent I'intersection possible des aspects ethniques avec des facteurs liés au genre, aux classes d'âge ou au statut social des défunts.

[49] Desiderio 2018, p. 73-76.

[50] Pellegrino et al. 2017, p. 231 ; Pellegrino \& Rizzo 2018, p. 155. 
Le processus de négociation lisible dans la culture matérielle concerne notamment les tombes de femmes et d'enfants. Un exemple supplémentaire est apporté par l'une des plus anciennes inscriptions étrusques de Pontecagnano : une séquence alphabétique gravée sur une œnochoé en impasto provenant de la tombe d'enfant 6034 du secteur Gaeta, datée du troisième quart du VII s. av. J.-C., (fig. 4B et 5). Or, la tombe d'enfant est superposée à la sépulture de la femme recroquevillée de la $\mathrm{T}$. 6032. La relation topographique souligne une ascendance étrangère dans le cadre d'une culture matérielle pleinement «locale », revendiquée par l'écriture. De plus, la volonté délibérée d'utiliser la langue étrusque est un indicateur de prestige. Elle peut représenter un instrument de légitimation, soulignant l'intégration politique au sein de la communauté [51].

\section{CONCLUSION}

Cet article n'a pas pour prétention de proposer une synthèse générale sur les phénomènes de mobilité et I'outil du genre. Nous avons cherché à mettre en perspective les différents cas d'étude, à les confronter - lorsque cela était possible - mais également à dégager de nouvelles pistes de recherche et contribuer au dossier en présentant un certain nombre d'observations. Les exemples abordés mettent I'accent sur I'exigence qu'il y aurait à s'interroger non seulement sur l'identité de genre, mais aussi sur celle du statut, I'identité de genre ne pouvant se suffire à elle-même. Le cas de la Campanie archaïque peut par ailleurs ouvrir des perspectives de recherche sur la mobilité, dépassant la seule question de l'identité ethnique. En effet, la diversité des comportements funéraires montre que les articulations sociales sont transversales aux composantes ethniques. À côté de formes probables de subordination, on observe également la présence de groupes allogènes qui élaborent des stratégies autonomes de représentation funéraire, alternatives ou compétitives par rapport aux autres noyaux funéraires. À Pithécusses, la construction du genre est un processus indissociable du groupe ethnique, mais elle se forge différemment, dans le temps, selon le statut et les choix culturels. La prise en compte de cette dynamique souligne alors la nécessité d'une approche, synchronique et diachronique, plus consciente des différences entre l'ensemble des catégories sociales et culturelles et au sein même de ces catégories. Par ailleurs, les comportements funéraires sont eux-mêmes si complexes qu'ils écartent I'utilisation de schémas interprétatifs uniques. À Pontecagnano, l'existence de formes hétérogènes de représentation laisse apparaître un processus actif d'intégration des composantes externes, inscrit dans une dimension communautaire qui, au moins en partie, dépasse le contrôle et la médiation des groupes locaux. La réorganisation d'époque orientalisante offre le cadre culturel, institutionnel et politique dans lequel s'inscrit l'intégration de larges segments de peuplement italique, qui contribuent à former, à différents titres, la communauté.

À l'issue de cette étude, le cas de la Campanie archaïque illustre, nous semble-t-il, la nécessité de rejeter des oppositions binaires et exclusives, celle de I'origine ethnique ou celle du genre. Seule une articulation intégrée des différentes dimensions sociales, tant au niveau des individus qu'au niveau des structures et des processus sociaux, peut permettre une meilleure compréhension des expériences de mobilité.

[51] CinquantaquatTro 2004-2005, p. 155-160; Cuozzo \& Pellegrino 2016, p. 55-56 ; Pellegrino et al. 2017, p. 232 ; Pellegrino \& Rizzo 2018, p. 156.

\section{REMERCIEMENTS}

Nous remercions Sandra Boehringer, Luca Cerchiai, Germaine Depierre et Carmine Pellegrino pour le temps qu'ils nous ont accordé et pour l'intérêt scientifique qu'ils ont manifesté pour notre projet. 
ATtI TARANTo à paraître a. La Magna Grecia nel Mediterraneo in età arcaica e classica, Atti del LVIII Convegno Internazionale di Studi sulla Magna Grecia, Taranto, 27-30 settembre 2018, Taranto, à paraître.

ATtr TARAnto à paraître $\boldsymbol{b}$. Donne di Magna Grecia. Visibilità, rappresentazione, ruoli, Atti del LIX Convegno Internazionale di Studi sulla Magna Grecia, Taranto, 26-28 settembre 2019, Taranto, à paraître.

Bartoloni, Gilda \& Nizzo, Valentino, 2005, « Lazio protostorico e mondo greco: considerazioni sulla cronologia relativa ed assoluta della terza fase laziale » dans Gilda Bartoloni \& Filippo Delpino (éd.), Oriente e Occidente: metodi e discipline a confronto. Riflessioni sulla cronologia dell'età del Ferro italiana, Roma, p. 409-436.

BeCKer, Marshall Joseph, 1995, « Human Skeletal Remains from the Pre-Colonial Greek Emporium of Pithekoussai on Ischia (NA): Culture Contact in Italy from the Early VIII to II Century B.C. », dans Neil Christie (éd.), Settlement and Economy in Italy 1500 B.C. to A.D. 1500, Oxford, p. 273-282.

DOI : $10.2307 / 507137$

BÉRARD, Reine-Marie, 2014, « Le métal et la parure : identité ethnique et identité de genre dans les nécropoles de Grande Grèce et de Sicile », Dialogues d'histoire ancienne Supplément 10, p. 145-169.

DOI : $10.3917 /$ dha.hs91.0145

BÉRARD, Reine-Marie, 2018, «Greek and Indigenous people: investigations in the cemeteries of Megara Hyblaea », dans Edward Herring \& Eóin O'Donoghue (éd.), Papers in Italian Archaeology VII. The Archaeology of Death, Proceedings of the Seventh Conference of Italian Archaeology held at the National University of Ireland, Galway, April 2016, Oxford, p. 48-57. BÉRARD, Reine-Marie, à paraître, « Tafonomia, determinazioni antropologiche del sesso e identità di genere », dans ATTI TARANTO à paraître $b$.

Boehringer, Sandra \& Sebillotte Cuchet, Violaine (dir.), 2011, Hommes et femmes dans l'Antiquité grecque et romaine. Le Genre : méthode et documents, Paris.

Boehringer, Sandra \& Sebillotte Cuchet, Violaine, 2013, « Vingt ans de réflexion : Mètis et le genre (1992-2012)», dans Sandra Boehringer \& Violaine Sebillotte Cuchet (éd.), Des femmes en action : L'individu et la fonction en Grèce antique, Paris - Athènes, p. 5-18.

DOI : $10.4000 /$ books.editionsehess.2825

Boenringer, Sandra, Grand-Clément, Adeline, Péré-Noguès, Sandra \& Sebillotte Cuchet, Violaine, 2020, «La base de données Eurykleia : Un outil au service d'une histoire mixte de I'Antiquité », dans Dossier : Des femmes qui comptent : Genre et participation sociale en Grèce et à Rome, Paris - Athènes, p. 19-37.

DOI : 10.4000/books. editionsehess.29797

Bonaudo, Raffaella, Cuozzo, Mariassunta, Mugrone, Eliana, Pellegrino, Carmine \& Serritella, Antonia, 2009, «Le necropoli di Pontecagnano: studi recenti », dans Raffaella Bonaudo, Luca Cerchiai \& Carmine Pellegrino (éd.), Tra Etruria, Lazio e Magna Grecia: indagini sulle necropoli, Paestum, p. 169-208.

Boufrier, Sophie, 2017, « Migrations et mobilités antiques : l'exemple des Grecs en Méditerranée » dans Dominique Garcia \& Hervé Le Bras (éd.), Archéologie des migrations, Paris, p. 153-166.

DOI : 10.3917/dec.garci.2017.01

Castiglioni, Maria Paola, 2019, La donna greca, Bologna.

CerchiaI, Luca, 1995, I Campani, Milano.

CerchiaI, Luca, 2013, « Mobilità nella Campania preromana: il caso di Pontecagnano », dans Giuseppe Maria Della Fina (éd.), Mobilità geografica e mercenariato nell'Italia preromana, Roma, p. 139-162.

CerchraI, Luca, 2017, « Integrazione e ibridismi campani: Etruschi, Opici, Euboici tra VIII e VII sec. a.C. », dans Ibridazione ed integrazione in Magna Grecia. Forme, modelli, dinamiche, Atti del XLIV Convegno Internazionale di Studi sulla Magna Grecia, Taranto, p. 221-242.

CerchiaI, Luca, 2018, « Società dei vivi, comunità dei morti: qualche anno dopo », Annali dell'Tstituto Orientale di Napoli, Sezione Archeologia e Storia Antica n.s. 25, p. 151-158.

CerchiaI, Luca, 2020, «La Campania in età arcaica, tra integrazione e conflitti », dans Maria Paola Castiglioni, Mariateresa Curcio \& Rachele Dubbini (éd.), Incontrarsi al limite: ibridazioni mediterranee nell'Italia preromana, Atti del convegno di Ferrara, 6-8 giugno 2019, Roma, p. 99-109. 
Cerchiat, luca, Cinquantaquattro, Teresa Elena \& Pellegrino, Carmine, 2013, « Dinamiche etnico-sociali e articolazioni di genere nell'Agro Picentino », dans Laura Guidi \& Maria Rosaria Pelizzari (éd.), Nuove frontiere per la Storia di genere, Salerno, p. 77-95.

Cinquantaquattro, Teresa Elena, 2004-2005, « Un nuovo alfabetario dall'Etruria campana: testimonianze di uso della scrittura a Pontecagnano nel periodo orientalizzante », Annali dell'Istituto Orientale di Napoli, Sezione Archeologia e Storia Antica n.s. 11-12, p. 155-165.

Cinquantaquattro, Teresa Elena, 2006-2007, « Rituale funerario e dinamiche di genere nel mondo indigeno della mesogaia campana: il caso di Avella », Annali dell'Istituto Orientale di Napoli, Sezione Archeologia e Storia Antica n.s. 13-14, p. 111-134.

Cinquantaquattro, Teresa Elena, 2012-2013, «La necropoli di Pithekoussai (scavi 1965-1967): variabilità funeraria e dinamiche identitarie, tra norme e devianze », Annali dell'Istituto Orientale di Napoli, Sezione Archeologia e Storia Antica n.s. 19-20, p. 31-58.

Cinquantaquattro, Teresa Elena, 2017, « Greci e Indigeni a Pithekoussai: i nuovi dati dalla necropoli di S. Montano (scavi 1965-1967) », Ibridazione ed integrazione in Magna Grecia. Forme, modelli, dinamiche, Atti del XLIV Convegno Internazionale di Studi sulla Magna Grecia, Taranto, p. 265-284.

Cinquantaquattro, Teresa Elena \& Cuozzo, Mariassunta, 2002, « Relazioni tra l'area daunia e medio-ofantina e la Campania. Nuovi apporti archeologici », dans Lisa Pietropaolo (éd.), Sformate immagini di bronzo. Il carrello di Lucera tra VIII e VII secolo a.C., Foggia, p. 127-138.

Coldstream, John Nicolas, 1994, «Prospectors and Pioneers: Pithekoussai, Kyme and Central Italy », dans Gocha Revazi Tsetskhladze \& Franco de Angelis (éd.), The Archaeology of Greek Colonisation, Oxford, p. 47-59.

Cuozzo, Mariassunta, 2000, « Orizzonti teorici e interpretativi, tra percorsi di matrice francese, archeologia post-processuale e tendenze italiane: considerazioni e indirizzi di ricerca per lo studio delle necropoli », dans Nicola Terrenato (éd.), Archeologia Teorica, Firenze, p. 323-360.

Cuozzo, Mariassunta, 2003, Reinventando la tradizione. Immaginario sociale, ideologie e rappresentazione nelle necropoli orientalizzanti di Pontecagnano, Paestum.

Cuozzo, Mariassunta, 2008, «Interpretazione delle necropoli e questioni di genere nell'archeologia italiana: il caso di Pontecagnano », dans Lourdes Prados Torreira \& Clara Ruiz López (éd.), Arqueología del género. 1er Encuentro Internacional en la UAM, Madrid, p. 105-138.

Cuozzo, Mariassunta \& Gurdr, Alessandro, 2013, Archeologia delle identità e delle differenze, Roma.

Cuozzo, Mariassunta \& Pellegrino, Carmine, 2016, « Rappresentazione e interpretazione: obiettivi e prospettive nella lettura delle necropoli. Alcune considerazioni sul significato degli oggetti iscritti », dans Marie-Laurence Haack (éd.), L'écriture et l'espace de la mort. Épigraphie et nécropoles à l'époque préromaine, Rome (Collection de l'École française de Rome 502), p. 39-57.

DOI : $10.4000 /$ books.efr.2704

Cuozzo, Mariassunta \& Pellegrino, Carmine, 2019, « Gentes e complessità archeologica: il caso di studio di Pontecagnano », dans Massimiliano Di Fazio \& Silvia Paltineri (éd.), La società gentilizia nell'Italia antica tra realtà e mito storiografico, Bari, p. 139-154.

D'Agostino, Bruno, 1985, «Società dei vivi, comunità dei morti: un rapporto difficile », Dialoghi di Archeologia n.s. 1, p. 47-58.

D'Agostino, Bruno, 1999, «Pitecusa e Cuma tra Greci e indigeni », La colonisation grecque en Méditerranée occidentale, Actes de la rencontre scientifique en hommage à Georges Vallet, Rome-Naples 15-18 novembre 1995, Rome (Collection de l'École française de Rome 251), p. 51-62.

D'Agostino, Bruno, 2011, «Pithecusa e Cuma nel quadro della Campania di età arcaica », Bullettino dell'Istituto Archeologico Germanico, Sezione romana 117, p. 35-53.

D'Ercole, Maria Cecilia, 2002, Importuosa Italiae litora. Paysage et échanges dans l'Adriatique méridionale à l'époque archaïque, Napoli.

DOI : 10.4000/books.pcjb.522

Desiderio, Anna Maria, 2018, « Material culture and ethnic identity: some case studies from Pontecagnano (first-second quarter of the seventh century BC) », dans Edward Herring \& Eóin O'Donoghue (éd.), Papers in Italian Archaeology VII. The Archaeology of Death, Proceedings of the Seventh Conference of Italian Archaeology held at the National University of Ireland, Galway, April 2016, Oxford, p. 70-78. 
Desiderio, Anna Maria \& Esposito, Arianna, 2020, « Migrations, mobilities and integrations in Campania (8th-7th centuries BC): trajectories and perspectives », dans Thibault Lachenal, Réjane Roure \& Olivier Lemercier (éd.), Demography and Migration. Population Trajectories from the Neolithic to the Iron Age, Oxford, p. 141-156.

Dommelen, Peter van, 2014, « Moving On: Archaeological Perspectives on Mobility and Migration », World Archaeology 46/4, p. 477-483.

DOI : $10.1080 / 00438243.2014 .933359$

Esposito, Arianna, 2018, « Rethinking Pithekoussai. Perspectives and current issues », dans Eric Gailledrat, Rosa Plana \& Michael Dietler (éd.), The Emporion in the Ancient Western Mediterranean. Trade and Colonial Encounters from the Archaic to the Hellenistic Period, Montpellier, p. 167-179.

Esposito, Arianna \& Pollini, Airton, 2018, « Have you said métissage or hybridization? A viewpoint from the graves », dans Edward Herring \& Eóin O’Donoghue (éd.), Papers in Italian Archaeology VII. The Archaeology of Death, Proceedings of the Seventh Conference of Italian Archaeology held at the National University of Ireland, Galway, April 2016, Oxford, p. 41-47.

Esposito, Arianna \& Zurbach, Julien, 2010, «Femmes indigènes et colons grecs : quelques observations », dans Pierre Rouillard (éd.), Portraits de migrants, portraits de colons, Paris, p. 51-70.

Gigante, Melania, Bondioli, Luca \& Sperduti, Alessandra, 2012-2013, « Di alcune sepolture della necropoli di Pithekoussai, Isola di Ischia - Napoli. Analisi preliminare dei resti odonto-scheletrici umani di VIII-VII sec. a.C. dagli scavi Buchner 1965-1967 », Annali dell'Istituto Orientale di Napoli, Sezione Archeologia e Storia Antica n.s. 19-20, p. 59-72.

Gigante, Melania, Warter, Viola, Muller, Wolfgang, Bondioli, Luca \& Sperduti, Alessandra, à paraître, «Anthropological Evidence of Multiethnicity in the First Greek Settlement in Italy. Strontium isotopic Analysis of the Skeletal Sample from the Necropolis of Pithekoussai, (Ischia VIII cent. BCE-III cent. CE) », Proceedings of the 86th Annual Meeting of The American Association of Physical Anthropologists (AAPA), à paraître $a$.

Gigante, Melania, Sperduti, Alessandra, Alhaique, Francesca, Fiore, Ivana, Cinquantaquattro, Teresa Elena, MulLeR, Wolfgang \& Bondrolr, Luca, à paraître, « Dinamiche demografiche e di mobilità dalla necropoli di Pithekoussai tra VIII e VI sec. a.C. Le evidenze scheletriche e isotopiche », Archeologia del cambiamento. Modelli, processi, adattamenti nella Preistoria e Protostoria, Atti della LVI Riunione Scientifica dell'Istituto Italiano di Preistoria e Protostoria (IIPP), Roma, 23-29 ottobre 2019, à paraître $b$.

Guzzo, Pier Giovanni, 2012, « Fibule e identità a Pithecusa », Archeologia classica LXIII, n.s. II, 2, p. 509-535.

Haicault, Monique, 2012, «Autour d'agency. Un nouveau paradigme pour les recherches de Genre », Rives méditerranéennes (1) 41, p. 11-24.

DOI : $10.4000 /$ rives.4105

Hodos, Thomas, 1999, «Intermarriage in the Western Greek colonies », Oxford Journal of Archaeology 18, p. 61-78. DOI : 10.1111/1468-0092.00071

IsaYev, Elena, 2017, Migration, Mobility and Place in Ancient Italy, Cambridge.

DOI : $10.1017 / 9781316440612$

JAmes, Sharon L. \& Dillon, Sheila (éd.), 2012, A Companion to Women in the Ancient World, Malden - Oxford - Chichester. Kelley, Olivia, 2012, «Beyond intermarriage: the role of the indigenous Italic population at Pithekoussai », Oxford Journal of Archaeology 31/3, p. 245-260.

DOI : 10.1111/j.1468-0092.2012.00388.x

KnAPP, Arthur Bernard \& Dommelen, Peter van, 2008, « Past Practices: Rethinking Individuals and Agents in Archaeology », Cambridge Archaeological Journal 18, p. 15-34.

DOI : $10.1017 / \mathrm{s} 0959774308000024$

MoAtrI, Claudia (dir.), 2004, La mobilité des personnes en Méditerranée de l'Antiquité à l'époque moderne. Procédures de contrôle et documents d'identification, Rome (Collection de l'École française de Rome 341).

MoATtI, Claudia, 2019, « Mobility in the Roman world: new concepts, new perspectives », dans Andrea Zerbini \& Justin Yoo (éd.), Migration, Diaspora and Identity in the Near East from Antiquity to the Middle Ages, London - New York, p. 15-25. DOI : 10.4324/9781351254762

MoATtI, Claudia, à paraître, «La mobilità mediterranea nella storiografia recente: modelli e prospettive di indagine », dans ATTI TARANTO à paraître $a$.

Nizzo, Valentino, 2007, Ritorno ad Ischia. Dalla stratigrafia della necropoli di Pithekoussai alla tipologia dei materiali, Napoli. Nrzzo, Valentino, 2015, Archeologia e antropologia della morte. Storia di un'idea, Bari. 
Nrzzo, Valentino, 2016a, « Cronologia versus Archeologia. L“ambiguo" scorrere del tempo alle soglie della "colonizzazione": i casi di Cuma e Pithekoussai », dans Valentino Nizzo, Lieve Donnelaan \& Gert-Jan Burgers (éd.), Contexts of early colonisation. (Contextualising early colonisation, volume II), Roma, p. 49-72.

Nizzo, Valentino, 2016b, « Per una stratigrafia dei rapporti sociali: parentela, rito, tempo e filtri funerari nella necropoli di Pithekoussai », Poleis e politeiai nella Magna Grecia arcaica e classica, Atti del LIII Convegno Internazionale di Studi sulla Magna Grecia, Taranto, p. 417-457.

Nrzzo, Valentino, 2021, «La 'costruzione' del paesaggio funerario: dinamiche di integrazione e filtri funerari nella necropoli di Pithekoussai », dans Reine-Marie Bérard (dir.), Il diritto alla sepoltura nel Mediterraneo antico, Roma, p. 33-77.

DOI : 10.4000/books.efr. 12788

Osborne, Robin, 2004, Greek History, London - New York.

Pellegrino, Carmine, 2015, « Pontecagnano e I'Agro Picentino: processi sociali, dinamiche territoriali e strutturazione urbana tra VIII e VII secolo a.C. », dans Giulia Saltini Semerari \& Gert-Jan Burgers (éd.), Early Iron Age communities of Southern Italy, Roma, p. 26-47.

DOI : $10.1017 /$ S1047759400074262

Pellegrino, Carmine, Rizzo, Carmelo \& Grimaldi, Tatiana, 2017, « Dall'Irpinia alla costa tirrenica: fenomeni di mobilità e integrazione in Campania tra VIII e VII secolo a.C. », dans Vincenzo Franciosi, Amedeo Visconti, Alessandra Avagliano \& Vittorio Saldutti (éd.), Appellati nomine lupi, Napoli, p. 207-273.

Pellegrino, Carmine \& Rizzo, Carmelo, 2018, « La necropoli occidentale di Pontecagnano. Segni di identità etnica nelle tombe dell'Orientalizzante antico e medio », Scienze dell'Antichità 24/2, p. 149-167.

PÉRÉ-Noguès, Sandra, 2008, « Recherches autour des "marqueurs funéraires" à travers l'exemple de quelques sépultures féminines de la nécropole du Fusco (Syracuse) », Pallas 76, p. 151-171.

ReIter, Samantha \& FreI, Karin Margarita, 2019, «Interpreting Past Human Mobility Patterns: A Model », European Journal of Archaeology 22/4, p. 454-469.

DOI : $10.1017 /$ eaa.2019.35

Saltini Semerari, Giulia, 2016, «Greek-Indigenous intermarriage: a gendered perspective », dans Lieve Donnelaan, Valentino Nizzo \& Gert-Jan Burgers (éd.), Conceptualising early colonization (Contextualising early colonisation, volume II), Brussel - Roma, p. 77-88.

Shepherd, Gillian, 1999, « Fibulae and Females: Intermarriage in the Western Greek colonies and the evidence from the cemeteries », dans Gocha Revazi Tsetskhladze (éd.), Ancient Greeks West and East, Leiden - Boston - Köln, p. 267-300. Shepherd, Gillian, 2005, «Dead men tell no tales: ethnic diversity in Sicilian colonies and the evidence of the cemeteries », Oxford Journal of Archaeology 24/2, p. 115-136.

DOI : 10.1111/j.1468-0092.2005.00228.x

ShePherd, Gillian, 2012, «Women in Magna Graecia », dans Sharon L. James \& Sheila Dillon (éd.), A Companion to Women in the Ancient World, Malden - Oxford - Chichester, p. 215-228.

ShePherd, Gillian, 2014, « Archaeology and Ethnicity: Untangling Identities in Western Greece », Dialogues d'histoire ancienne Supplément 10, p. 115-143.

ThébAud, Françoise, 1998, Écrire l'histoire des femmes, Paris. 